\title{
Three-arm robot-assisted thoracoscopic surgery for locally advanced N2 non-small cell lung cancer
}

\author{
Xinghua Cheng, Chongwu Li, Jia Huang, Peiji Lu, Qingquan Luo \\ Shanghai Chest Hospital, Shanghai Pulmonary Tumor Medical Center, Shanghai 200030, China \\ Correspondence to: Dr. Qingquan Luo. Shanghai Chest Hospital, Shanghai Pulmonary Tumor Medical Center, 241 West Huai-Hai Road, Shanghai \\ 200030, China. Email: qingquanluo@hotmail.com.
}

Submitted Nov 05, 2018. Accepted for publication Nov 15, 2018.

doi: $10.21037 /$ jtd.2018.11.94

View this article at: http://dx.doi.org/10.21037/jtd.2018.11.94

\section{Introduction}

Video-assisted thoracoscopic surgery (VATS) is now recognized as the standard treatment of early staged lung cancer due to improved postoperative recovery and equivalent oncological efficacy as compared to the conventional open approach $(1,2)$. However, its use in locally advanced lung cancer has not been adopted by the majority of thoracic surgeons. Technical challenges and the concern on insufficient lymph node dissection are the main obstacles to the application of VATS in this group of patients (3).

Robot-assisted thoracoscopic surgery (RATS) as a rapidly developing, next-generation, minimally invasive technique, has recently gained increasing popularity. The robotic platform has several technical advantages over VATS, including three-dimensional operative view, intuitive use of the robotic hands, and more flexibility of the instruments. It therefore has the potential to surpass VATS in handling more difficult cases $(4,5)$. Although the feasibility of RATS in locally advanced non-small cell lung cancer (NSCLC) has only been evaluated in limited retrospective studies (4-6), it is believed this technique may promote the use of minimally invasive surgery in these patients in the near future.

Since 2009, over 1,000 lung resections have been performed by our team using the da Vinci S robot system $(7,8)$. Here, we report a representative case accompanied by a surgical video to share our experience in managing locally advanced N2 NSCLC using a three-arm RATS approach.

\section{Operative techniques}

\section{Initial evaluation}

After admission, all patients undergo standard evaluation for operability of the tumor. If presence of stage III (N2) disease is suspected, patients usually require a whole-body PET-CT scan and an endobronchial ultrasound guided fine-needle aspiration biopsy (EBUS-FNA) for staging. These cases will then be discussed by a multi-disciplinary team for appropriate treatment modalities.

\section{Clinical vignette}

The presenting case was a 65 -year-old gentleman with a heavy smoking history (with 800 cigarette-years, who quit 2 weeks prior to the admission). He also had histories of hypertension, atrial fibrillation and coronary artery stent implantation. Contrast chest CT showed a right upper lobe mass (Figure 1) with enlarged hilar (10R) and mediastinal lymph nodes (2R and 4R). Preoperative lung functions were moderately impaired (FEV1\% and DLCO \% were $80.7 \%$ and $82.7 \%$, respectively). The patient was diagnosed as clinical T1N2M0 adenocarcinoma by CT-guided biopsy and PET-CT, but he refused to take EBUS-TBNA or inductive chemoradiotherapy due to the fear of disease progression, so surgery was planned. As an eligible case, this patient was enrolled in a randomized trial comparing RATS to open surgery in stage II-IIIA NSCLC that is currently running in our institution, and was assigned to the RATS 

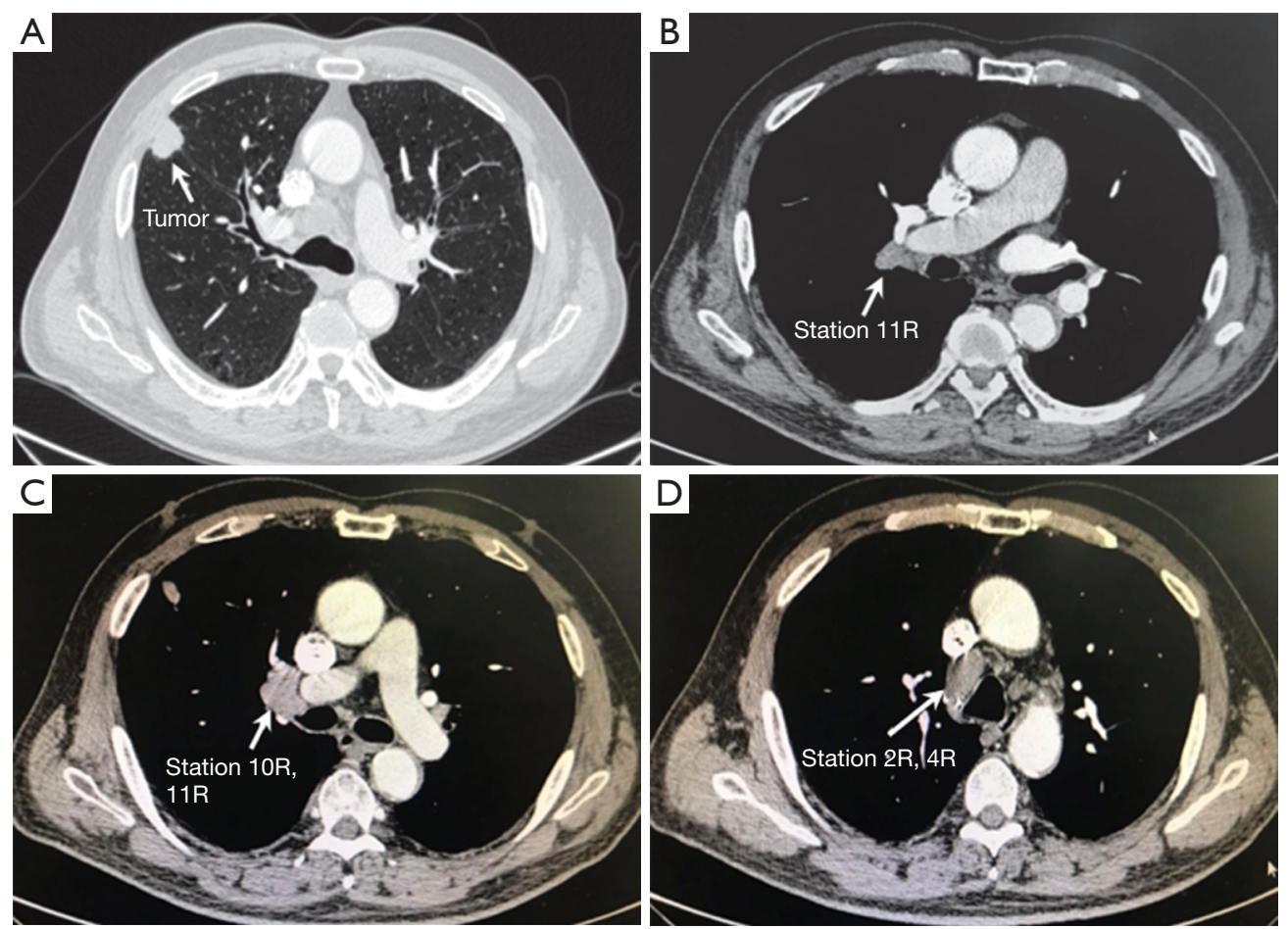

Figure 1 Contrast computed tomography of the presenting case. A $2.3 \mathrm{~cm} \times 2 \mathrm{~cm}$ solid mass was located in the right upper lobe (A) with enlarged station 11 (B), station 10 (C), stations 2 and 4 (D) lymph nodes.

group before the operation. A right upper lobectomy with systematic lymph node dissection was performed. Postoperative recovery was uneventful and the patient was discharged 4 days after the surgery. The final pathology confirmed the diagnosis (adenocarcinoma, pT1N2M0, stage IIIa) so adjuvant chemotherapy was prescribed. At 12-month follow-up, there was no sign of recurrence.

\section{Operation room setting and incisions (Figure 2)}

After intubation, the patient is placed in the lateral decubitus position with his/her head straight toward the robot. Firstly, a camera port $(12 \mathrm{~mm})$ is placed in the $7^{\text {th }}$ (for upper lobes) or $8^{\text {th }}$ (for lower lobes) intercostal space at the post-axillary line. A $30^{\circ}$ camera is then used to explore the pleural cavity. If there is no severe adhesion or metastasis and the lung deflates well, 2 other arm ports $(8 \mathrm{~mm})$ are then introduced both anteriorly and posteriorly to the camera port. The arm ports are usually at the same level to, or slightly above the camera port, and an 8-10 cm inter-port distance is required to avoid instrument interference. A utility incision of $3 \mathrm{~cm}$ is then placed in the $4^{\text {th }}$ intercostal space in front of the anterior border of the latissimus dorsi. Once all trocars are in position, we start docking the robot which normally takes 3-5 minutes. For the three-arms approach, a fenestrated clamp is inserted through robotic arm 1 (surgeon left hand) and a cautery hook is inserted through arm 2 (surgeon right hand). For the smoothness of the operation, we prefer not to change these instruments during the surgery. Sponge forceps, staplers and clips are introduced through the utility incision by a bedside assistant.

\section{Surgical steps}

In most cases, the operation starts from the dissection of the subcarinal lymph node stations and then proceeds in a posterior to anterior "single direction" manner to avoid repeated lung flipping.

For right upper lobectomy in cases like this (Figure 3), the lower lobe is first retracted anteriorly. Subcarinal pleural is opened from the upper border of the inferior pulmonary vein to the azygos vein along the esophagus and the intermediate bronchus. All lymph nodes (station 7 and 8) in the subcarinal region are removed in an "en-bloc" fashion and both the left and right bronchus and pericardium are exposed clearly after dissection. 

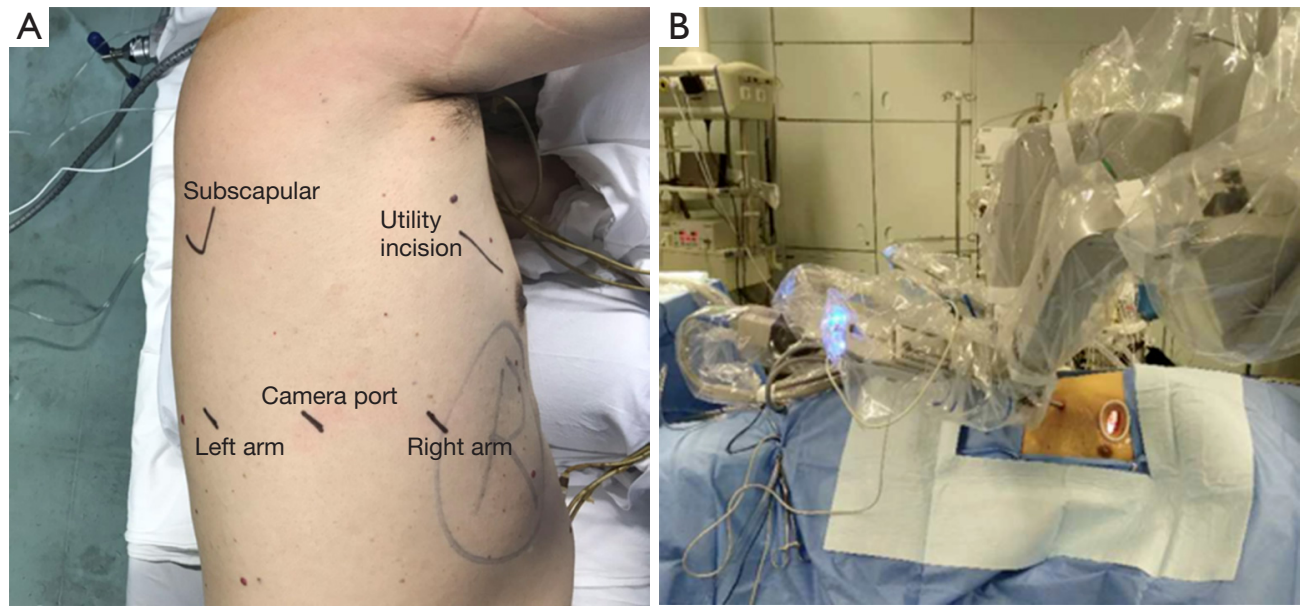

Figure 2 Ports positions of the three-arm approach (A). The camera port for right upper lobectomy is placed in the $7^{\text {th }}$ intercostal space at the post-axillary line and two-arm ports are $8-10 \mathrm{~cm}$ away from the camera ports. For docking, the patient is placed in the lateral decubitus position straight to the robot (B).

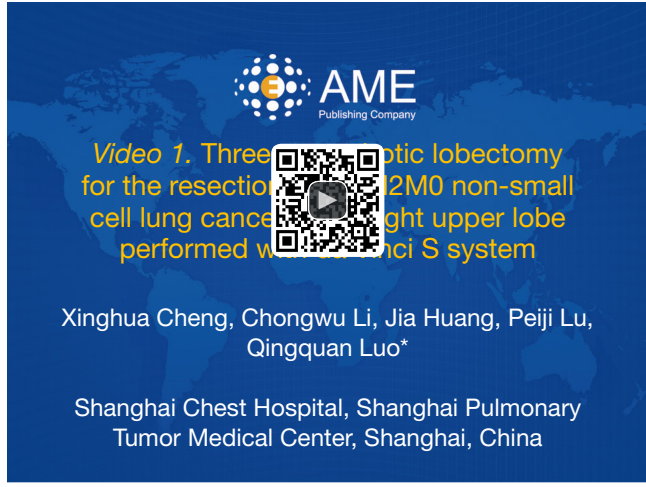

Figure 3 Three-arm robotic lobectomy for the resection of cT1N2M0 non-small cell lung cancer in the right upper lobe performed with da Vinci S system (9).

Available online: http://www.asvide.com/article/view/29198

Posterior hilar tissue between the intermediate bronchus and the upper lobe bronchus is then released to facilitate upper lobe bronchus mobilization. Posterior hilar lymph nodes (station 10) and interlobar lymph nodes (station 11) are removed during this process. In most cases, especially for those with suspected nodal involvement, it is necessary to divide the oblique fissure for better exposure and complete removal of all interlobar nodes. The upper lobe bronchus is further mobilized and divided by a stapler.

After completion of the bronchus, the upper lobe is retracted to the apex by clamping the bronchus stump, and the hilar lymph nodes (station 10) are carefully removed.
During this process, the posterior side of the truncus branch and the superior pulmonary vein are also dissected. The upper lobe is then retracted posteriorly to expose the anterior hilum. Both the superior truncus branch and superior pulmonary vein are further isolated anteriorly. A tunnel was made from the space underneath the inferior border of the vein to the interlobar space for the division of horizontal fissure by staplers. After completion of the horizontal fissure, the arteries and veins to the upper lobe are divided together by staplers and the upper lobe is retrieved using a specimen bag.

For the dissection of tracheal lymph nodes, mediastinal pleura is opened from the upper margin of the azygos vein to the level of the subclavian artery along the superior vena cava and the trachea. Injury to the veins and the right recurrent laryngeal nerve should be carefully avoided. For complete removal of station 4R lymph nodes, it is usually necessary to dissect the region underneath the azygos vein. Normally, the azygos vein can be kept intact. In the presenting case, however, as the $4 \mathrm{R}$ lymph nodes were significantly enlarged, for better exposure and dissection, the azygos vein was divided by a stapler. The $2 \mathrm{R}$ and $4 \mathrm{R}$ lymph nodes were then removed in an en-bloc fashion.

\section{Comments}

Locally advanced NSCLC represent a mixed group of cancers and local treatments are critical for these patients. Although reported oncological outcomes vary in different 
studies (5-year overall survival 30-45\%) (10-12), radical surgery is still considered the most effective modality for these patients. However, whether surgery can truly bring clinical benefits to this group of patients largely depends on surgical morbidity and completeness of the resection $(10,13)$. Particularly, in patients with nodal involvement, complete dissection of lymph nodes has been shown to be related with a reduced local recurrence rate and a better survival (13).

Patients with stage III NSCLC are generally more fragile and usually have systemic treatment prior to the surgery. Therefore, thoracoscopic surgery, which reduces surgical morbidity and accelerates post-operative recovery $(1,2)$, should be a better option for these patients than open surgery. However, dissection in these patients, especially those with enlarged nodes, is more challenging using VATS; thus, safety and completeness of the surgery may be compromised. These concerns impede the diffusion of VATS in patients with more advanced disease.

With better vision, more maneuverability and more static movement, the ability of RATS in fine manipulation and lymph node dissection has been greatly improved. It has been shown that robotic technique may be associated with higher nodal upstaging rate than VATS in patients with clinical stage I NSCLC $(14,15)$. Evidence on the application of RATS in locally advanced NSCLC is also accumulating. Cerfolio et al. recently reported a 5-year survival of $62 \%$ for patients with Stage IIIa NSCLC after robotic lobectomy and $73 \%$ for those with N2 disease (5). In another multi-institutional retrospective study by Veronesi et al., resections of pstage IIIIa lung cancer by RATS yielded similar oncological results (3-year overall survival $61 \%$ and local recurrence rate $8.5 \%$ ) to the open approach (4). The conversion rate was $9.9 \%$, which is lower than that reported for VATS previously $(6,16)$. Despite these encouraging early results, further validation by prospective studies is still required.

In the current article, we showed a three-arm robotic lobectomy for clinical N2 NSCLC. This technique has been verified in our institution and the operational outcomes are satisfactory (7). Previously, Veronesi et al. described a fourarm approach for the resection of locally advanced NSCLC and the specimens were removed from an additional utility incision $(17,18)$. The reasons why we used the three-arm approach are: (I) additional arms and instruments add extra cost to the total surgical expense in our hospital; (II) only 4 incisions are made including the utility incision; and (III) no dislodgment of the instrument arms is needed in this procedure. Nevertheless, as robotic stapler currently is not available in China, it needs to be noted that all staplers are inserted from limited angles through the utility incision. To avoid excessive tension during division, vessels must be sufficiently mobilized; thus, we attempted to transect the bronchus first in order to facilitate lung retraction.

In conclusion, radical resection of locally advanced lung cancer by RATS is feasible and the ability to handle difficult lymph nodes is significantly enhanced. With the progression of robotic technology, the future of minimally invasive technique in patients with more advanced NSCLC is promising.

\section{Acknowledgements}

Funding: This work was supported by the Shanghai Hospital Development Center Grant (SHDC12016113) and National Natural Science Foundation of China (81502480).

\section{Footnote}

Conflicts of Interest: The authors have no conflicts of interest to declare.

Informed Consent: Written informed consent was obtained from the patient for publication of this manuscript and any accompanying images.

\section{References}

1. Bendixen M, Jorgensen OD, Kronborg C, et al. Postoperative pain and quality of life after lobectomy via video-assisted thoracoscopic surgery or anterolateral thoracotomy for early stage lung cancer: a randomised controlled trial. Lancet Oncol 2016;17:836-44.

2. Yan TD, Black D, Bannon PG, et al. Systematic review and meta-analysis of randomized and nonrandomized trials on safety and efficacy of video-assisted thoracic surgery lobectomy for early-stage non-small-cell lung cancer. J Clin Oncol 2009;27:2553-62.

3. Vannucci F, Gonzalez-Rivas D. Is VATS lobectomy standard of care for operable non-small cell lung cancer? Lung Cancer 2016;100:114-9.

4. Veronesi G, Park B, Cerfolio R, et al. Robotic resection of Stage III lung cancer: an international retrospective study. Eur J Cardiothorac Surg 2018;54:912-9.

5. Cerfolio RJ, Ghanim AF, Dylewski M, et al. The longterm survival of robotic lobectomy for non-small cell lung cancer: A multi-institutional study. J Thorac Cardiovasc 
Surg 2018;155:778-86.

6. Park BJ, Yang HX, Woo KM, et al. Minimally invasive (robotic assisted thoracic surgery and video-assisted thoracic surgery) lobectomy for the treatment of locally advanced non-small cell lung cancer. J Thorac Dis 2016;8:S406-13.

7. Huang J, Li J, Li H, et al. Continuous 389 cases of Da Vinci robot-assisted thoracoscopic lobectomy in treatment of non-small cell lung cancer: experience in Shanghai Chest Hospital. J Thorac Dis 2018;10:3776-82.

8. Zhao X, Qian L, Lin H, et al. Robot-assisted lobectomy for non-small cell lung cancer in china: initial experience and techniques. J Thorac Dis 2010;2:26-8.

9. Cheng X, Li C, Huang J, et al. Three-arm robotic lobectomy for the resection of cT1N2M0 non-small cell lung cancer in the right upper lobe performed with da Vinci S system. Asvide 2018;5:936. Available online: http:// www.asvide.com/article/view/29198

10. Albain KS, Swann RS, Rusch VW, et al. Radiotherapy plus chemotherapy with or without surgical resection for stage III non-small-cell lung cancer: a phase III randomised controlled trial. Lancet 2009;374:379-86.

11. Pless M, Stupp R, Ris HB, et al. Induction chemoradiation in stage IIIA/N2 non-small-cell lung cancer: a phase 3 randomised trial. Lancet 2015;386:1049-56.

12. Zheng D, Ye T, Hu H, et al. Upfront surgery as first-line therapy in selected patients with stage IIIA non-small cell lung cancer. J Thorac Cardiovasc Surg 2018;155:1814-22.e4.

13. Izbicki JR, Passlick B, Pantel K, et al. Effectiveness of radical systematic mediastinal lymphadenectomy in patients with resectable non-small cell lung cancer: results of a prospective randomized trial. Ann Surg 1998;227:138-44.

14. Wilson JL, Louie BE, Cerfolio RJ, et al. The prevalence of nodal upstaging during robotic lung resection in early stage non-small cell lung cancer. Ann Thorac Surg 2014;97:1901-6; discussion 1906-7.

15. Boffa DJ, Kosinski AS, Paul S, et al. Lymph node evaluation by open or video-assisted approaches in 11,500 anatomic lung cancer resections. Ann Thorac Surg 2012;94:347-53; discussion 353.

16. Gonfiotti A, Bongiolatti S, Bertolaccini L, et al. Thoracoscopic lobectomy for locally advanced-stage nonsmall cell lung cancer is a feasible and safe approach: analysis from multi-institutional national database. J Vis Surg 2017;3:160.

17. Veronesi G, Novellis P, Difrancesco O, et al. Robotic assisted lobectomy for locally advanced lung cancer. J Vis Surg 2017;3:78.

18. Park BJ, Flores RM, Rusch VW. Robotic assistance for video-assisted thoracic surgical lobectomy: technique and initial results. J Thorac Cardiovasc Surg 2006;131:54-9.
Cite this article as: Cheng X, Li C, Huang J, Lu P, Luo Q. Three-arm robot-assisted thoracoscopic surgery for locally advanced N2 non-small cell lung cancer. J Thorac Dis 2018;10(12):7009-7013. doi: 10.21037/jtd.2018.11.94 